CLINICAL STUDIES / ETUDES CLINIQUES

\title{
IMAGERIE DES FISTULES ARTERIO-VEINEUSES DURALES A DRAINAGE VEINEUX PERI
} -MEDULLAIRE

\section{IMAGING OF SPINAL DURAL ARTERIOVENOUS FISTULAS}

MELHAOUI Adyl 1

EL QUESSAR Abdeljalil ${ }^{1}$

EL HASSANI Moulay Rachid ${ }^{1}$

CHAKIR Noureddine 1

JIDDANE Mohammed ${ }^{1}$

1. Service de neuroradiologie, Rabat, Royaume du Maroc

E-Mail Contact - MELHAOUI Adyl : adymelh (at) yahoo (dot) com

Mots clés : Fistule durale, Rachis, IRM, Angiographie

Key words : Dural fistula, Spine, MRI, Angiography

\section{RESUME}

Les fistules durales rachidiennes à drainage veineux périmédullaire constituent une pathologie d'individualisation récente. C'est les plus fréquentes des malformations vasculaires vertébromédullaires, elles se présentent sous la forme d'une myélopathie spastique progressive chez un sujet de plus de 40 ans. A partir d'une étude rétrospective des données cliniques et explorations radiologiques d'une série de 11 cas en IRM et artériographie médullaire, nous avons recensé l'ensemble des signes radiologiques qui orientent le diagnostic.

L'IRM a montré un hypersignal T2 centromédullaire de façon constante ainsi que des vaisseaux périmédullaires pathologiques. L'angiographie médullaire reste l'examen clé pour le diagnostic. Elle a permit de montrer dans dix cas le shunt artério-veineux ainsi que ses afférences artériels et son retour veineux, permettant de planifier le traitement. Huit patients ont été pris en charge chirurgicalement avec exclusion de la fistule suivie d'une amélioration clinique. L'imagerie joue aussi un rôle important dans le suivi postthérapeutique. L'IRM permet de mettre en évidence la disparition progressive des anomalies initiales, signe de l'efficacité du traitement. Les fistules artério-veineuses à drainage veineux périmédullaire sont une pathologie qui reste encore méconnue. La connaissance de tous ces éléments d'imagerie est fondamentale pour un diagnostic et une prise en charge précoces, seuls garants d'une bonne évolution après traitement.

\section{SUMMARY}

Spinal dural arteiovenous fistulas are the most frequent type of spinal vascular malformations. Usual clinical presentation is of progressive myelopathy in an adult above the age of 40 years.

Based on a retrospective study of clinical and radiologic data (MRI and Angiography) concerning a series of 11 cases, we have collected all the radiologic finding that led to the diagnosis. In all cases MRI showed spinal cord abnormalities, consistent in a central T2 hypersignal and perimedullary flow voids. The angiography remains the gold standard method for diagnosis. It can show the exact location of the shunt unless it is thrombosed, as in one of our patients, thus allowing accurate treatment planning. Eight patients underwent a surgical treatment allowing the closure of the fistula that resulted in significant neurological improvement. Postoperative MRI shows the results of the treatment. The disappearance of preoperative abnormalities is a good indicator for the efficiency of the treatment.

Spinal dural arteriovenous fistulas is still an underdiagnosed condition, the good knowledge of these typical imaging findings is mandatory for an early diagnosis and treatment. 


\section{INTRODUCTION}

Individualisées par KENDALL et LOGUE en 1977 (13) puis par MERLAND et coll en 1980 (18), les fistules durales rachidiennes à drainage veineux périmédullaires (FAVDPM) sont les plus fréquentes des anomalies artério-veineuses intra-rachidiennes.

Elles sont rares et souvent méconnues, elles surviennent de façon prédominante chez l'homme de quarante à soixante ans et entraînent l'apparition d'une myélopathie chronique par hyperpression veineuse. En l'absence de traitement, l'évolution se fait lentement vers une paraplégie définitive.

En nous appuyant sur l'étude de 11 observations cliniques et sur une revue de la littérature nous proposons une mise au point sur l'ensemble des anomalies radiologiques permettant d'évoquer le diagnostique, nous évaluerons également la place de l'imagerie dans le traitement et le suivi post thérapeutique de ces patients.

\section{MATERIEL ET METHODES}

II s'agit d'une étude rétrospective des données cliniques et radiologique de 11 cas de fistules durales rachidiennes à drainage veineux péri-médullaires, explorées au service de neuroradiologie de l'hôpital des spécialités O.N.O de Rabat entre 1997 et 2002. Tous les patients ont bénéficié d'une IRM avec des séquences multiplanairesT1, T2 et T1 avec injection de gadolinium ainsi que d'une angiographie médullaire. Une Myélographie a été réalisée dans 1 cas. Le traitement à été chirurgical dans 8 cas. Deux patients ont refusé une prise en charge chirurgicale. Une embolisation a été réalisée dans un cas.

\section{RESULTATS}

Nos observations portent sur onze cas de FAVDPM colligées entre 1997 et 2002 sur un ensemble de 16 MAV médullaires diagnostiquées la même période, représentant $68 \%$ des malformations artérioveineuses rachidiennes traitées au sein du service.

Tous nos patients sont de sexe masculin. L'âge moyen est de 50 ans avec des extrêmes entre 37 et 65 ans. Le tableau clinique était fait dans tous les cas d'une paraparésie spastique à début insidieux, le délai d'évolution avant diagnostic variait de 3 mois à 3 ans avec une moyenne de 1 an et trois mois (Tableau 1). Les signes cliniques de début sont souvent à type d'algies dorsales ou lombaires, parfois cruralgies (cas $n^{\circ} 6$ ), des troubles génito-sphinctériens frustes sont souvent retrouvés à cette phase de début.

Au moment du diagnostique tous nos patients avaient un déficit moteur qui allait d'une discrète paraparésie permettant la marche (cas $\left.n^{\circ} 1,2,9\right)$ à une paraplégie complète confinant le malade à la chaise roulante (cas $\left.n^{\circ} 2\right)$. Tous les malades présentaient une hypertonie spastique, avec syndrome pyramidal dans trois cas $\left(n^{\circ} 1\right.$, 2 et 5 ).

Les troubles sensitifs étaient inconstants à type de paresthésies dans 2 cas ( $n^{\circ} 1$ et 2$)$, anesthésie en selle dans un cas $\left(n^{\circ} 1\right)$ et hypoesthésie à niveau sous ombilical dans un cas $\left(n^{\circ} 2\right)$.

Concernant les résultats des explorations radiologiques (Tableau 2): Les clichés standards de débrouillage réalisés systématiquement à la phase de début retrouvaient des signes d'arthrose fréquents à cet âge. L'IRM réalisée chez tous les patients, montrait dans tous les cas des anomalies intracanalaires à type de :

- Elargissement du cône médullaire dans 11 cas / 11 plus ou moins marqué.

- Hypersignal T2 dans tous les cas, son étendue moyenne est de 6 niveaux.

- Hyposignal T1 retrouvé dans 8 cas.

- Veines périmédullaires dilatées : retrouvées dans tous les cas, étendue en dorsolombaire dans 9 cas, en dorsal uniquement dans un cas ( $\left.n^{\circ} 4\right)$, et arrivant jusqu'au niveau cervical dans un cas $\left(n^{\circ} 9\right)$. Les veines étaient dans tous les cas postéro-médullaires, dans 5 cas elles étaient associées à des veines antérieures dilatées.

Dans deux cas les patients ont bénéficié de deux examens IRM, la première exploration étant jugée insuffisante par le médecin traitant. ( $\mathrm{N}^{\circ} 1$ et 2 ) L'angiographie médullaire a été réalisée dans tous les cas, l'artère spinale antérieure (ASA) n'a pu être visualisée que dans 4 cas elle naissait respectivement en D8, D10 et D12 (2cas).

Dans dix cas une FAVDPM a pu être visualisée, dans un cas il s'agissait d'une fistule thrombosée $\left(n^{\circ} 6\right)$. Les niveaux intéressés allaient de D4 à L4. Dans 6 cas la fistule siégeait à gauche, et dans 3 cas à droite. Le drainage veineux se faisait de façon variable, uniquement ascendante dans un cas $\left(n^{\circ} 3\right)$ ou associée à un drainage descendant dans le reste des cas.

La prise en charge a été chirurgicale dans 9 cas, avec abord direct de la fistule et exclusion de la veine de 
drainage. Dans un cas $\left(n^{\circ} 11\right)$ une embolisation par un matériel particulaire a été tentée avec bonne évolution initiale mais récidive de la symptomatologie au bout de six mois, signant une réperméabilisation, le malade a été repris chirurgicalement.

Les suites opératoires ont été dans tous les cas simples, il ne fut pas noté de cas d'aggravation après chirurgie. L'évolution post-opératoire a été marquée par une amélioration de la symptomatologie clinique portant essentiellement sur les troubles moteurs (cas $n^{\circ} 3,4,7,8,9$ et 11), une simple stabilisation des lésions dans le cas $n^{\circ} 1$.

La surveillance radiologique post opératoire a consisté en une artériographie de contrôle montrant l'exclusion de la fistule, une IRM médullaire à 6 mois dans 2 cas ( $n^{\circ} 4$ et 11) montrant la disparition des anomalies IRM. Le recul moyen est de 2 ans.

\section{DISCUSSION}

Les fistules durales rachidiennes se définissent comme un shunt artèrioveineux microscopique de 40 à $140 \mu$ (10), situé en extra-médullaire, dans l'épaisseur même de la dure-mère. Il s'agit d'une communication directe entre une ou plusieurs artérioles radiculo-méningées et une veine radiculaire unique se drainant vers les veines périmédullaires. C'est un shunt à faible débit, le plus souvent situé en regard du trou de conjugaison ou sur le trajet d'une racine postérieure. Toute fistule est constamment associée à des perturbations du drainage veineux médullaire. Le drainage veineux se fait de manière descendante vers les veines de la queue de cheval ou ascendante vers la fosse cérébrale postérieure. Elles représentent le type I selon la classification de Spetzler (23).

Leur étiopathogénie reste incertaine, leur âge tardif de survenue leur fait attribuer une origine acquise (6). Leur mécanisme d'expression clinique est en rapport avec une ischémie médullaire d'origine veineuse traduction de la gêne au retour veineux accompagnant le développement de la FAVDPM (13).

Les malformations vasculaires médullaires restent une pathologie rare (25). Actuellement les fistules durales sont reconnues comme étant les malformations vasculaires médullaires les plus fréquentes (18) DJINDJIAN sur une série de 296 MAVs médullaires, retrouvait 35\% de FAVDPM et BRUNERAU (5) sur une série de 140 MAVs, retrouvait $28 \%$ de FAVDPM. Dans notre série elles constituent $68 \%$. Le sex-ratio se situe entre $6 / 1$ et 9/1 selon les séries (4-48). Dans notre série tous les patients sont de sexe masculin. L'âge de survenue de la pathologie se situe dans la cinquième décennie avec un âge moyen de 55 ans (4- 7), l'âge moyen de nos patients était de 50 ans. Ces données épidémiologiques : prédominance masculine et survenue à la cinquième décennie sont caractéristiques des FAVDPM.

Le tableau clinique typique associe un début fruste avec un délai diagnostique souvent supérieur à 1 an comme dans notre série (24-22). II constitue un tableau de myélopathie progressive associant des troubles moteurs intéressant les membres inférieures le plus souvent, troubles accompagnés de troubles sensitifs et sphinctériens. Cette symptomatologie est souvent asymétrique aggravée par l'effort et la manœuvre de Valsalva (14). Non traité il évolue vers une paraplégie définitive. Des cas d'aggravation aigue ont été rapportés (22).

Leur Topographie est essentiellement dorsale et dorsolombaire de rares cas de localisation cervicale ou sacrée ont été rapportée (15-20).

Apport de la radiologie

L'IRM médullaire est l'examen à réaliser en première intention devant toute suspicion de FAVDPM. (Figure 1) L'examen doit comporter une exploration dans les trois plans et comporter une injection de Gadolinium. La fistule elle-même n'est pas visible étant de taille microscopique, I'IRM permet de montrer les signes de présemption qui sont :

- Un élargissement du cône médullaire : signe constant. mais non spécifique. Dans notre série il est retrouvé dans $100 \%$ des cas.

- Des anomalies de signal intramédullaires: (Figure 2)

- Un hypersignal T2 centromédullaire : c'est le signe le plus sensible, il est d'étendue variable, pouvant s'étendre jusqu'au niveau cervical comme le patient $n^{\circ} 9 . \quad(2)$ - Un hyposignal T1 : élément inconstant retrouvé surtout dans les formes chroniques. - Un rehaussement du signal médullaire après injection de Gadolinium aurait une valeur pronostic selon LARSSON.

- Un hyposignal médullaire périphérique en T2 qui serait un signe spécifique de myélopathie par hyperpression veineuse selon HURST-GROSSMAN (11).

- Des anomalies de signal périmédullaires à type de structures vides de signal serpigineuses hypointenses sur les séquences T1 et T2 et se rehaussant après injection de Gadolinium, correspondant aux veines de drainage péri médullaires dilatées.

Les séquences plus récentes d'angiolRM médullaires permettraient d'affiner cette analyse radiologique (3) 
en orientant vers le niveau de la fistule préparant ainsi l'angiographie qui reste le seul examen permettant d'affirmer de manière certaine le diagnostique et le siège de la FAVDPM.

L'exploration angiographique doit comporter l'opacification de toutes les branches radiculomédullaire, l'alimentation de la fistule pouvant être multiétagée (23). Elle doit également identifier la naissance de l'ASA. Elle permet de visualiser le shunt, les pédicules nourriciers et les veines de drainage qui sont essentiellement postérieures.

Le shunt est de très petite taille. II est indiqué par un brutal changement de calibre entre l'artère nourricière et la veine de drainage. II se projette habituellement, sur l'incidence de face, en dessous ou en dedans du pédicule vertébral.

Un signe diagnostique spécifique serait le retard du retour veineux de l'ASA de l'ordre de 15 à 20 secondes (4). Cette angiographie peut rester négative comme dans notre cas $n^{\circ} 6$ pour cause de thrombose de la fistule ou en raison d'une maladie athéromateuse gênant l'exploration. (12)

Le diagnostique différentiel se pose sur le plan clinique avec une compression médullaire lente et toutes les causes de syndrome de la queue de cheval et de radiculalgie lombaire. Sur le plan de l'imagerie il faudrait différencier les anomalies de signal observées en IRM d'une tumeur intramédullaire, d'une syringomyélie, d'une atteinte médullaire de sclérose en plaque ou encore d'accident vasculaire médullaire. L'artériographie permettra de distinguer les FAVDPM par rapport aux autres malformations vasculaires rachidiennes et médullaires.

Le but du traitement est d'exclure définitivement le shunt artérioveineux pour permettre la normalisation de la pression dans le réseau veineux périmédullaire. Comme dans notre série le traitement chirurgical est simple (1) et permet la guérison dans la majorité des cas (19). Le traitement endovasculaire comporte un risque d'aggravation neurologique et de reperméabilisation comme dans notre cas $n^{\circ} 7$. Une de ses contre indications est la naissance de la fistule à partir d'une des branches de l'ASA. (8) Le suivi clinique et radiologique permet de constater une amélioration dans la majorité des cas. Les anomalies de signal IRM vont progressivement disparaître (9), l'hypersignal T2 intramédullaire et les anomalies vasculaires régressent dés le premier mois suivant le traitement. L'élargissement du cône médullaire peut évoluer vers une atrophie. Après traitement il est recommandé de réaliser une IRM de contrôle entre 3 à 6 mois, puis une IRM à un an post-traitement. L'artériographie de contrôle post-opératoire est réservée aux cas de non amélioration après 3 mois du traitement à la recherche d'une autre fistule ou d'une reperméabilisation (21).

Le pronostic reste lié essentiellement à la durée d'évolution des signes cliques (17) malheureusement sous nos contrées le fait que cette pathologie reste encore méconnue ajouté à la faible disponibilité des examens IRM et explorations neuroradiologiques invasives ont tendance à allonger le délai diagnostic.

\section{CONCLUSION}

Les FAVDPM sont une pathologie rare mais invalidante. Elles sont accessibles à un traitement curatif d'autant plus efficace que le diagnostique est porté précocement. Toute myélopathie progressive chez un patient de plus de 40 ans doit faire évoquer le diagnostique et conduire à la réalisation rapide d'une IRM médullaire complétée d'une artériographie médullaire pour le diagnostic de certitude. Ceci permettra un traitement dans les meilleurs délais. 
Tableau $n^{\circ} 1$ : Résumé des données cliniques

\begin{tabular}{|c|c|c|c|c|c|c|c|c|}
\hline Cas & Age & Sexe & Délai & $\begin{array}{l}\text { Syndrome } \\
\text { pyramidal }\end{array}$ & $\begin{array}{l}\text { Troubles } \\
\text { sensitifs }\end{array}$ & $\begin{array}{l}\text { Déficit } \\
\text { moteur }\end{array}$ & Troubles GS & Autres \\
\hline$N^{\circ} 1$ & $\begin{array}{r}42 \\
\text { ans }\end{array}$ & M & 2 ans & $\begin{array}{l}\text { Hypertonie } \\
\text { spastique } \\
\text { pyramidale } \\
\text { Babinski } \\
\text { bilatérale }\end{array}$ & $\begin{array}{l}\text { Paresthésies } \\
\text { anesthésie en } \\
\text { selle }\end{array}$ & $\begin{array}{c}\text { CIM } \\
\text { Parararésie }\end{array}$ & $\begin{array}{l}\text { Impériosité } \\
\text { Impuissance }\end{array}$ & Lombalgies \\
\hline $\mathrm{N}^{\circ} 2$ & $\begin{array}{r}65 \\
\text { ans }\end{array}$ & M & $\begin{array}{c}1 \text { an et } \\
\text { demi }\end{array}$ & $\begin{array}{l}\text { Hypertonie } \\
\text { Babinski } \\
\text { bilatéral }\end{array}$ & $\begin{array}{c}\text { paresthésies des } \\
2 \mathrm{MI} \\
\text { niveau sensitif }\end{array}$ & Paraplégie & $\begin{array}{c}\text { Dysurie } \\
\text { Constipation }\end{array}$ & \\
\hline $\mathrm{N}^{\circ} 3$ & $\begin{array}{r}50 \\
\text { ans }\end{array}$ & M & 1 an & $\begin{array}{l}\text { Hypertonie } \\
\text { spastique }\end{array}$ & $\begin{array}{c}\text { Pas de troubles } \\
\text { sensitifs }\end{array}$ & $\begin{array}{l}\text { Paraparésie } \\
\text { CIM }\end{array}$ & Impériosité & $\begin{array}{c}\text { Dorso- } \\
\text { Lombalgies }\end{array}$ \\
\hline $\mathrm{N}^{\circ} 4$ & $\begin{array}{r}50 \\
\text { ans }\end{array}$ & M & 1 an & $\begin{array}{l}\text { Hypertonie } \\
\text { spastique }\end{array}$ & $\begin{array}{l}\text { Pas de troubles } \\
\text { sensitifs }\end{array}$ & Paraparésie & $\begin{array}{l}\text { Incontinence } \\
\text { urinaire }\end{array}$ & Dorsalgies \\
\hline$N^{\circ} 5$ & $\begin{array}{r}37 \\
\text { ans }\end{array}$ & M & 6 mois & $\begin{array}{l}\text { Syndrome } \\
\text { pyramidale }\end{array}$ & $\begin{array}{l}\text { Pas de troubles } \\
\text { sensitifs }\end{array}$ & Paraparésie & - & - \\
\hline $\mathrm{N}^{\circ} 6$ & $\begin{array}{c}44 \\
\text { ans }\end{array}$ & M & 10 mois & Spastique & $\begin{array}{l}\text { Pas de troubles } \\
\text { sensitifs }\end{array}$ & Paraparésie & $\begin{array}{l}\text { Incontinence } \\
\text { Impuissance }\end{array}$ & Cruralgies \\
\hline $\mathrm{N}^{\circ} 7$ & $\begin{array}{r}45 \\
\text { ans }\end{array}$ & M & 18 mois & Hypertonie & $\begin{array}{c}\text { Pas de troubles } \\
\text { sensitifs }\end{array}$ & Paraparésie & $\begin{array}{l}\text { Incontinence } \\
\text { urinaire }\end{array}$ & - \\
\hline $\mathrm{N}^{\circ} 8$ & $\begin{array}{r}54 \\
\text { ans }\end{array}$ & M & 1 an & $\begin{array}{c}\text { Irritation } \\
\text { pyramidal }\end{array}$ & $\begin{array}{l}\text { Pas de troubles } \\
\text { sensitifs }\end{array}$ & Paraparésie & Tb mictionnels & - \\
\hline$N^{\circ} 9$ & $\begin{array}{r}47 \\
\text { ans }\end{array}$ & M & 3 mois & Spastique & $\begin{array}{l}\text { Pas de troubles } \\
\text { sensitifs }\end{array}$ & Paraparésie & Tb mictionnels & - \\
\hline$N^{\circ} 10$ & $\begin{array}{r}61 \\
\text { ans }\end{array}$ & M & 3 ans & Spastique & $\begin{array}{c}\text { Paresthésies des } \\
\mathrm{Ml}\end{array}$ & CIM & Incontinence & - \\
\hline $\mathrm{N}^{\circ} 11$ & $\begin{array}{r}59 \\
\text { ans }\end{array}$ & M & $\begin{array}{l}\text { 1an et } \\
\text { demi }\end{array}$ & Spastique & $\begin{array}{l}\text { Pas de troubles } \\
\text { sensitifs }\end{array}$ & Paraparésie & $\begin{array}{l}\text { Incontinence } \\
\text { Impuissance }\end{array}$ & - \\
\hline
\end{tabular}


Tabeau N²2 : Résumé des données radiologiques.

\begin{tabular}{|c|c|c|c|c|c|c|c|c|}
\hline \multirow[t]{3}{*}{ Cas } & IRM & & & & & Artériographie & & \\
\hline & Moelle & & & & $\begin{array}{l}\text { Anomalies } \\
\text { vasculaires }\end{array}$ & $\begin{array}{l}\text { Fistule artério } \\
\text { veineuse }\end{array}$ & & \\
\hline & $\begin{array}{l}\text { Cône } \\
\text { élargi }\end{array}$ & НуроТ1 & $\begin{array}{l}\text { Hyper T2 } \\
\text { étendue }\end{array}$ & Gado & $\begin{array}{l}\text { Présence et } \\
\text { étendue }\end{array}$ & siége & $\begin{array}{l}\text { Niveau } \\
\text { afférence }\end{array}$ & $\begin{array}{l}\text { Drainage } \\
\text { veineux }\end{array}$ \\
\hline 1 & oui & non & $\begin{array}{c}\text { oui } \\
\text { D8 D11 }\end{array}$ & non & oui & $\begin{array}{l}\text { Ant/ } \\
\text { post }\end{array}$ & D4 gauche & $\begin{array}{l}\text { Ascendant } \\
\text { postérieur }\end{array}$ \\
\hline 2 & oui & non & $\begin{array}{c}\text { oui } \\
\text { D2 à L1 }\end{array}$ & oui & $\begin{array}{l}\text { oui modérée } \\
\text { D4 à L2 }\end{array}$ & post & L2 Droite & $\begin{array}{l}\text { Ascendant } \\
\text { postérieur }\end{array}$ \\
\hline 3 & oui & oui & $\begin{array}{c}\text { oui } \\
\text { D8 à L1 }\end{array}$ & non & $\begin{array}{l}\text { oui } \\
\text { DL }\end{array}$ & $\begin{array}{l}\text { Ant/ } \\
\text { post }\end{array}$ & D6 gauche & Ascendant \\
\hline 4 & oui & oui & $\begin{array}{c}\text { oui } \\
\text { D7 à L1 }\end{array}$ & oui & $\begin{array}{c}\text { oui } \\
\text { Dorsale }\end{array}$ & Post & D9 gauche & $\begin{array}{c}\text { Ascendant et } \\
\text { descendant post }\end{array}$ \\
\hline 5 & oui & non & modéré & oui & $\begin{array}{c}\text { oui } \\
\text { D3 à L1 }\end{array}$ & $\begin{array}{l}\text { Ant } \\
\text { Post }\end{array}$ & D7 gauche & $\begin{array}{l}\text { Ascendant et } \\
\text { descendant }\end{array}$ \\
\hline 6 & oui & oui & oui & non & oui & Post & $\begin{array}{c}\text { Fistule } \\
\text { thrombosée }\end{array}$ & - \\
\hline 7 & oui & oui & $\begin{array}{c}\text { oui } \\
\text { D5-L4 }\end{array}$ & non & oui & Post & D4 gauche & Ascendant post \\
\hline 8 & oui & oui & $\begin{array}{c}\text { oui } \\
\text { D8-D9 à } \\
\text { L1 }\end{array}$ & non & $\begin{array}{l}\text { oui } \\
\text { D7 à L2 }\end{array}$ & $\begin{array}{l}\text { Ant } \\
\text { Post }\end{array}$ & D11 Droite & $\begin{array}{l}\text { Ascendant } \\
\text { Descendant }\end{array}$ \\
\hline 9 & oui & oui & $\begin{array}{c}\text { oui } \\
\text { discret } \\
\text { D9 à D12 }\end{array}$ & non & $\begin{array}{l}\text { oui } \\
\text { Cervico- } \\
\text { Dorso- } \\
\text { Lombaire }\end{array}$ & Post & D10 Droite & $\begin{array}{c}\text { Postérieur et } \\
\text { antérieur } \\
\text { Ascendant } \\
\text { intracrânien et } \\
\text { descendant S1 }\end{array}$ \\
\hline 10 & oui & oui & $\begin{array}{c}\text { oui } \\
\text { D5 à L1 }\end{array}$ & non & $\begin{array}{l}\text { oui } \\
\text { Dorsal }\end{array}$ & Post & D9 gauche & Ascendant \\
\hline 11 & oui & oui & $\begin{array}{c}\text { oui } \\
\text { D7 à L4 }\end{array}$ & non & $\begin{array}{c}\text { oui } \\
\text { Dorsolombaire }\end{array}$ & Post & L1 Droite & $\begin{array}{l}\text { Ascendant et } \\
\text { descendant }\end{array}$ \\
\hline
\end{tabular}


Figure $n^{\circ}$ 1: Conduite des exploration radiologiques devant un tableau de myélopathie progréssive

Syndromemedullaire (symptonatologieangue ou cluonque

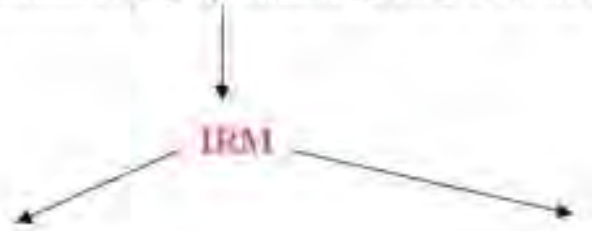

Pathologie

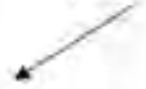

Pathologie tumorale

Infectieuse ou

Degenenative typique
Hypersignal T2 du cône et/ou

Augmentation de volumedu còne et/o11

Vaisseaur anommax intracanalaures Ou paravertebratr:

tRM ou séquences dynamiques

Artêrograplice

Figure 1

Conduite des explorations radiologiques devant une myélopathie progressive.
Aitériogaphie si clinique évocatace 


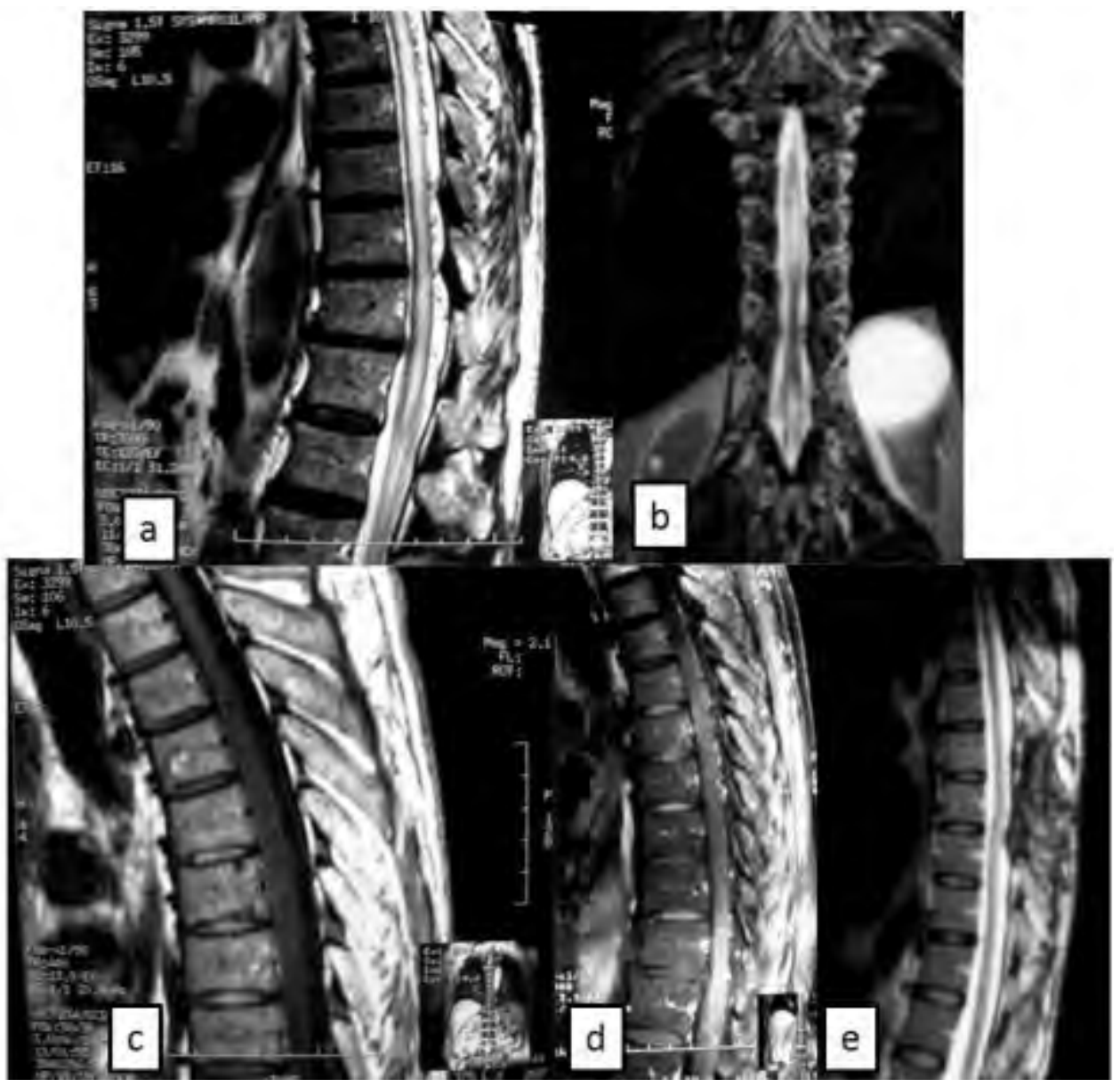

Figure 2

Aspect IRM des FAVDPM : hyper signal intramédullaire ascendant à partir d'un cône médullaire élargi visible en séquence T2 sagittale (a) et T2 coronale (b). Aspect hyposignal en intramédullaire sur les séquences sagittales T1 (c).Les anomalies de signal rétromédullaires visibles en T2 (a) se rehaussent après injection de contraste (d). 3 mois après la chirurgie on note une régression des anomalies médullaire (e). 


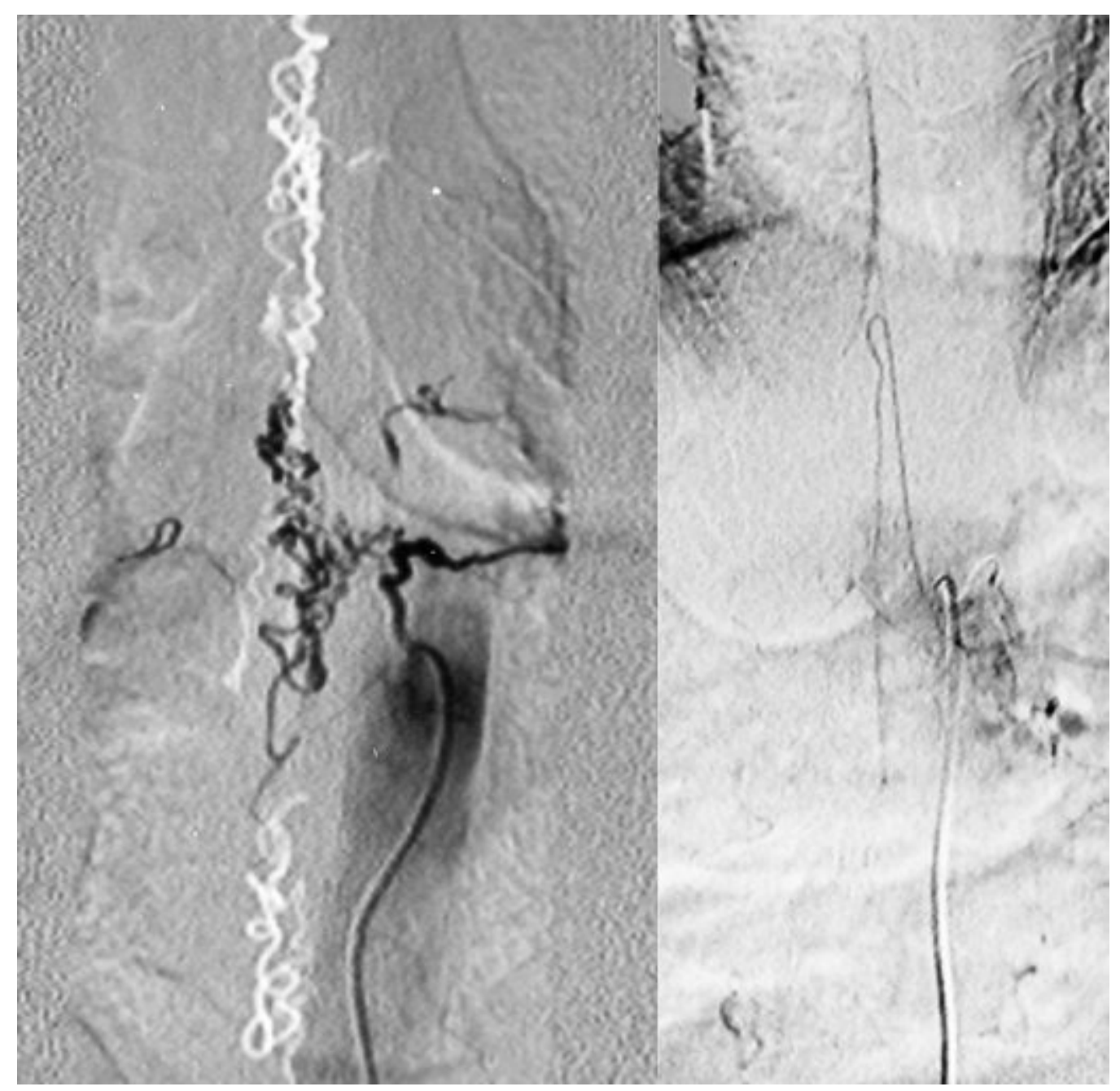

Figure 3

Artériographie médullaire du patient $n^{\circ} 5$ montrant le shunt au niveau D7 gauche avec un drainage veineux ascendant et descendant. L'artère d'ADAMKIEWICZ nait au niveau D12 gauche. 


\section{REFERENCES}

1. AFSHAR JK, DOPPMAN JL, OLDFIELD EH. Surgical interruption of intradural draining vein as curative treatment of spinal dural arteriovenous fistulas. J Neurosurg 1995 Feb;82(2):196-200

2. ASSOULINE E, GELBERT F, DORMONT D, REIZINE D, MERLAND JJ. MRI study of dural arteriovenous fistulae draining into the external spinal veins. Seven cases.J Neuroradiol. 1988;15(1):1-12.

3. BOWEN BC, FRASER K, KOCHAN JP, PATTANY PM, GREEN BA, QUENCER RM. Spinal dural arteriovenous fistulas: evaluation with MR angiography. AJNR Am J Neuroradiol 1995 NovDec;16(10):2029-43.

4. BRADAC GB, DANIELE D, RIVA A, BRACCHI M, STURA G, RICCIO A, PAGNI CA. Spinal dural arteriovenous fistulas: an underestimated cause of myelopathy. Eur Neurol 1994;34(2):87-94

5. BRUNERAU L, GOBIN YP, MEDER JF, et al Intracranial dural arteriovenous fistula with spinal venous draining: relation between clinical presentation and angiographic findings. AJNR 17:15491554,1996

6. GOBIN YP, HOUDART E, CASASCO A, MERLAND JJ. Endovascular therapy for arteriovenous malformations and fistulae in the spinal cord. Semin Intervent Radiol 1993; 10:227-242

7. GRANDIN C, DUPREZ T, STROOBANDT G, LATERRE EC, MATHURIN P. Spinal dural arteriovenous fistula: an underdiagnosed disease? Acta Neurol Belg 1997 Mar;97(1):17-21

8. HALL WA, OLDFIELD EH, DOPPMAN JL. Recanalization of spinal arteriovenous malformations following embolization. J Neurosurg 1989; 70:714-720

9. HORIKOSHI T, HIDA K, IWASAKI Y, ABE H, AKINO M. Chronological changes in MRI findings of spinal dural arteriovenous fistula. Surg Neurol. 2000 Mar;53(3):243-9.

10. HOUDART E, CHAPOT R, BOISSONET H, MERLAND JJ. Fistules artérioveineuses durales rachidiennes Encycl Méd Chir, Neurologie, 17-490-B-20, 2000, 4p.

11.HURST RW, GROSSMAN RI. Peripheral spinal cord hypointensity on T2-weighted MR images: a reliable imaging sign of venous hypertensive myelopathy. AJNR Am J Neuroradiol 2000 Apr;21(4):781-6

12.INCI S, BERTAN V, CILA A. Angiographically occult epidural arteriovenous fistula of the craniocervical junction. Surg Neurol. 2002 Mar; 57(3):167-73

13.KENDALL BE, LOGUE V. Spinal epidural angiomatous malformations draining into intrathecal veins Neuroradiology $1977 ; 13: 181-189$

14.Khurana VG, Perez-Terzic CM, Petersen RC, Krauss WE. Singing paraplegia: a distinctive manifestation of a spinal dural arteriovenous fistula. Neurology 2002 Apr 23;58(8):1279-81

15.KOHNO M, TAKAHASHI H, IDE K, ISHIJIMA B, YAMADA K, NEMOTO S. A cervical dural arteriovenous fistula in a patient presenting with radiculopathy. Case report. J Neurosurg. $1996 \mathrm{Jan}$; 84(1):119-23.

16.LARSSON EM, DESAI P, HARDIN CW, STORY J, JINKINS JR. Venous infarction of the spinal cord resulting from dural arteriovenous fistula: MR imaging findings. AJNR Am J Neuroradiol. $1991 \mathrm{Jul}-$ Aug;12(4):739-43.

17.LEV N, MAIMON S, RAPPAPORT ZH, MELAMED E. Spinal dural arteriovenous fistulae-a diagnostic challenge. Isr Med Assoc J. 2001 Jul;3(7):492-6.

18.MERLAND JJ, RICHE MC. CHIRAS J. Les fistules artérioveineuses intracanalaires, extramédullaires à drainage veineux médullaire. J Neuroradiology 1980; 7: 271-320.

19.MOURIER KL, GELBERT F, REY A, ASSOULINE E, GEORGE B, REIZINE D, MERLAND JJ, COPHIGNON J. Spinal dural arteriovenous malformations with perimedullary drainage. Indications and results of surgery in 30 cases. Acta Neurochir (Wien). 1989;100(3-4):136-41

20.O'BRIEN WT, SCHWARTZ ED, HURST RW, SINSON G. Spinal dural arteriovenous fistula with supply from sacral arteries. Surg Neurol. 2001 Sep;56(3):175-6

21.PRESTIGIACOMO CJ, NIIMI Y, SETTON A, BERENSTEIN A. Three-dimensional rotational spinal angiography in the evaluation and treatment of vascular malformations. AJNR Am J Neuroradiol. 2003 Aug; 24(7):1429-35.

22.ROSENBLUM B, OLDFIELD EH, DOPPMAN JL, DI CHIRO G. Spinal arteriovenous malformations: a comparison of dural arteriovenous fistulas and intradural AVM's in 81 patients. J Neurosurg. 1987 Dec;67(6):795-802

23.SPETZLER RF , DETWILER PW, et al Modified Classification of spinal cord vascular lesions. Journal of Neurosurgery ( Spine 2). $2002 ; 96: 145-156$.

24.SYMON L, KUYAMA H, KENDALL B. Dural arteriovenous malformations of the spine: Clinical 
features and surgical results in 55 cases. J Neurosurg 1984;60:238-47.

25.WITYK RJ. Dural arteriovenous fistula of the spinal cord: an uncommon cause of myelopathy Seminars in neurology, 1996 , Vol.16, No.1: 27-32 